Check for updates

Cite this: Chem. Sci., 2017, 8, 5616

Received 25th January 2017

Accepted 2nd June 2017

DOI: $10.1039 / \mathrm{c} 7 \mathrm{sc} 00373 \mathrm{k}$

rsc.li/chemical-science

\title{
Imaging of formaldehyde in plants with a ratiometric fluorescent probe $\uparrow$
}

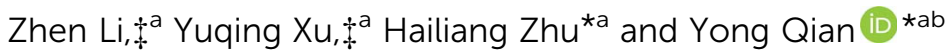

The fluorescence monitoring of formaldehyde in real environmental samples and live plant tissues is of great importance for physiological and pathological studies. However, there is a lack of suitable chemical tools to directly trace and measure the formaldehyde activity in bio-systems, and developing effective and, in particular, selective sensors for mapping formaldehyde in live tissues still remains a great challenge. Here, we demonstrate for the first time that the ratiometric fluorescence monitoring of formaldehyde in live plant tissues is achieved with a newly developed ratiometric fluorescent probe, FAP, which effectively eliminated interference from other comparative analytes. Live tissue analyses reveal that FAP can potentially detect exogenous and endogenous formaldehyde in live Arabidopsis thaliana tissues, exposing a potential application for biological and pathological studies of formaldehyde.

\section{Introduction}

Formaldehyde (FA), the simplest of the aldehydes with the formula $\mathrm{H}-\mathrm{CHO}$, has been widely used as an important precursor to many industrial materials and chemical compounds. ${ }^{1}$ On the other hand, it has also been classified as a highly toxic compound to living organisms, because its strong interactions with proteins, nucleic acids and other biomolecules will lead to the inactivation of their biological activities. ${ }^{2}$ For instance, excess exposure of exogenous FA to the human body will lead to a significant health threat and cause many diseases, ${ }^{3-6}$ including various cancers, heart disorders, diabetes, etc. Indeed, it has been regarded as a suspected carcinogen and well-known human toxicant. In plants, FA pollution is a widespread problem that can affect plant growth and development, causing a significant decrease of the productivity and quality in agriculture. ${ }^{7}$ But, interestingly, intracellular FA can be endogenously formed by normal physiological processes and spontaneously removed by complex metabolism using detoxification mechanisms. It has been demonstrated that glutathionedependent FA dehydrogenase (FALDH), as a key enzyme of FA metabolism, plays an essential role in the uptake and detoxification of FA in Arabidopsis plants. $^{8}$ Thus, the concentration of FA in living organisms is generally maintained in a dynamic state of equilibrium ranging from low to high millimolar

${ }^{a}$ State Key Laboratory of Pharmaceutical Biotechnology, School of Life Sciences, Nanjing University, No. 163 Xianlin Road, Nanjing 210023, China. E-mail: yongqian@nju.edu.cn

${ }^{b}$ College of Chemistry and Materials Science, Nanjing Normal University, No. 1 Wenyuan Road, Nanjing, 210046, China. E-mail: yongqian@njnu.edu.cn

$\dagger$ Electronic supplementary information (ESI) available. See DOI: $10.1039 / \mathrm{c} 7 \mathrm{sc} 00373 \mathrm{k}$

\$ These authors contributed equally to this work. levels. ${ }^{8-11}$ For instance, a small pool of FA $\left(0.1-10 \mu \mathrm{mol} \mathrm{g}^{-1}\right.$ fresh weight) was identified in the intermediate of the $\mathrm{C} 1$ metabolism of plants. ${ }^{12,13}$ The primary sources of FA in plants are caused by the processes of methanol oxidation, dissociation of 5,10methylene-THF, glyoxylate decarboxylation and other oxidative demethylation reactions. ${ }^{14}$ Exogenous FA can also be absorbed and incorporated into the metabolism of plants. ${ }^{7,15,16}$ As a result, phytoremediation has been extensively applied for the removal of FA from the polluted environment. However, the biological roles of FA and the mechanism of FA metabolism in plants have not yet been clearly defined, ${ }^{\mathbf{1 4}}$ and they are still challenging to study due to the lack of tools that can map the distribution of FA in live tissues. Thus, in order to understand the biological and pathological roles of FA well, it is crucial to develop sensitive methods or chemical tools for tracing and identifying FA in environmental samples and live plant tissues.

Coupled with fluorescence microscopy techniques, fluorescent imaging using small molecular fluorescent probes to study biological species in living bio-systems has attracted great interest owing to its high spatiotemporal resolution, noninvasiveness, high sensitivity, and excellent selectivity, ${ }^{17-28}$ which can offer the opportunity to monitor FA directly and easily in different biological contexts. However, the effective and, in particular, selective mapping of FA activity in live tissues with fluorescent probes still remains a great challenge. Recently, only a few fluorescent turn-on probes have been reported for monitoring FA. $^{17-21,29}$ Some could be successfully used for imaging FA in living cells, others were primarily applied for the in vitro determination of FA in water. These intensity-based fluorescent probes may have a number of limitations imposed by the fluorescent turn-on response at a single detection window, which means it is difficult to obtain accurate information when testing the FA concentration. Moreover, 
these turn-on responses can also vary depending on the experimental conditions, including the probe concentration, excitation intensity, temperature, and instrument efficiency. ${ }^{30}$ To minimize most of the aforementioned artificial and environmental factors, an attractive approach for the determination of FA in environmental samples and live tissues is developing ratiometric fluorescent probes, due to their quantitative tracking ability and self-calibration effect. ${ }^{23,31,32}$ So far, there is only one reported ratiometric fluorescent probe for monitoring $\mathrm{FA}^{33}$ but its relatively low excitation in the UV range and its short emission wavelength might easily be overwhelmed by the tissue's auto-fluorescence background, thereby limiting the probe's further application in live tissue. In addition, fluorescent probes that have the ability to image inside live plant tissues to provide relevant information on endogenous FA are particularly attractive, but no such probe has been reported to date. Therefore, developing novel ratiometric probes that can be applied for the in vivo imaging of FA with highly sensitive and selective responses is critically important and there is strong demand. We herein present the development and application of a FA probe, FAP (Scheme 1), a new ratiometric fluorescent sensor that can selectively report formaldehyde in real water samples and enable tracking of the endogenous FA activity in live tissues of Arabidopsis thaliana for the first time.

\section{Results and discussion}

To design this new FA ratiometric fluorescent probe, we focus on the 2-aza-Cope rearrangement reaction, ${ }^{34}$ as illustrated in Scheme 1, where the starting imine intermediate can be formed by the selective capture of FA with a homoallylamino group, and it will further undergo a simple rearrangement and hydrolysis process to yield an aldehyde product, ${ }^{17,18}$ potentially resulting in obvious changes of the fluorescence properties. Thus, we envisioned that the homoallylamino group can possibly serve as the FA selective response site. Subsequently, in order to obtain a ratiometric response, we chose a well-known $N, N$ dimethylquinolin-6-amine as the fluorescent chromophore because of its exhibited excellent photophysical and chemical properties, ${ }^{35,36}$ which has been used in the design of other reported ratiometric fluorescent probes as a fluorescence reporter. We anticipated that the free probe would exhibit a relatively short emission wavelength owing to the internal

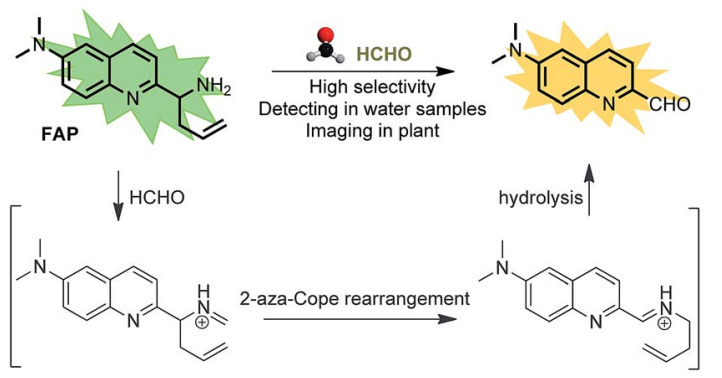

Scheme 1 The structure of FAP and the proposed ratiometric sensing mechanism for monitoring formaldehyde (FA). charge transfer (ICT) effect between the $N, N$-dimethyl group and the 2-position homoallylamine of the chromophore. Upon treatment with FA, however, the homoallylamino group would change into an aldehyde group via specifically activated processes, which would then cause an increase in the electron withdrawing ability, thereby inducing the red shift of the fluorescence emission spectrum of the probe and producing a ratiometric response for FA. Based on these design considerations, we then synthesized the fluorescent probe, FAP, in three steps starting from commercially available $N 1, N 1$ dimethylbenzene-1,4-diamine (Scheme 2), and the structures of the target compounds were fully characterized by ${ }^{1} \mathrm{H}$ and ${ }^{13} \mathrm{C}$ NMR spectroscopy and mass spectrometry (see the ESI $\dagger$ ).

As expected, the homoallylamino modification on FAP leads to relatively short absorbance and fluorescence. The free FAP $(20 \mu \mathrm{M})$ exhibited two absorption peaks at $254 \mathrm{~nm}$ and $353 \mathrm{~nm}$, and one broad emission peak at $495 \mathrm{~nm}$. However, the incubation of $20 \mu \mathrm{M}$ FAP with $1 \mathrm{mM}$ FA resulted in a remarkable red shift of the absorbance signals ( $23 \mathrm{~nm}$ and $76 \mathrm{~nm}$, respectively). As shown in Fig. 1a, two obvious absorption bands with peaks at $277 \mathrm{~nm}$ and $429 \mathrm{~nm}$ newly emerged, and the intensities of the original peaks at $254 \mathrm{~nm}$ and $353 \mathrm{~nm}$ simultaneously decreased; meanwhile, accompanying the reaction of FAP with FA, the emission wavelength was also obviously shifted $(75 \mathrm{~nm})$, affording an important enhancement of the fluorescence emission band at $570 \mathrm{~nm}$ and a significant decrease at $495 \mathrm{~nm}$ (Fig. 1b). These in vitro responses of FAP validate the previous design strategy and confirm that FAP is a good sensor for in vitro formaldehyde determination. In order to elaborate the reaction mechanism, we then monitored the reactions between FAP and FA using HPLC and analysed the reactions using MS and IR spectroscopy. The reactions with different incubation times showed clean conversion, and a new peak $(\mathrm{m} / \mathrm{z}=201.1)$, identified as the product with the expected mass of the aromatic aldehyde (3), began to appear after $1 \mathrm{~h}$ of incubation (Fig. S1 and $\mathrm{S} 2 \dagger)$. The reactions of different FA concentrations also showed a gradual decrease in the probe peak and a corresponding increase in the product peak with increasing FA concentration (Fig. S2 $\dagger$ ). In addition, a signal of the freshly formed -CHO- group was also clearly observed in the IR spectrum after treatment with FA (Fig. S3†). Taken together, these findings show that the formation of the aldehyde is indeed promoted by reacting with FA.

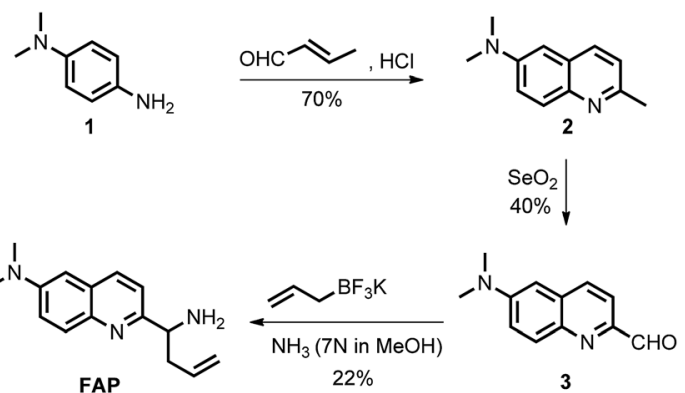

Scheme 2 Synthesis of the ratiometric fluorescent probe, FAP, for monitoring formaldehyde (FA). 

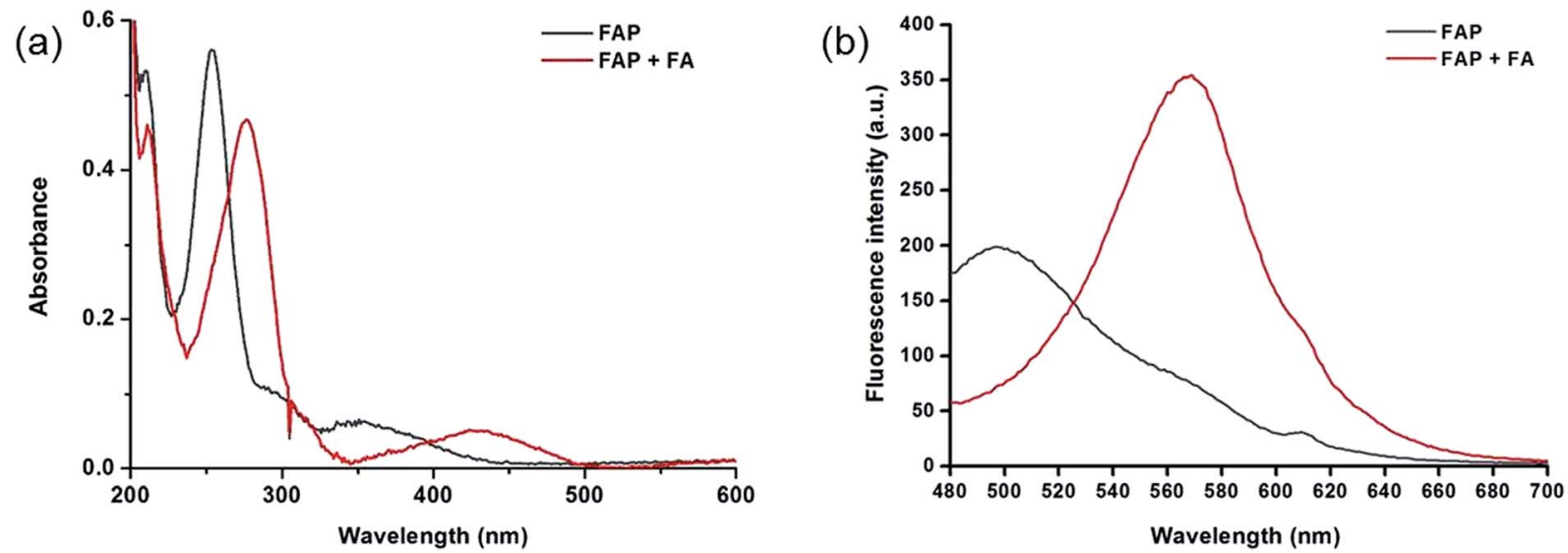

Fig. 1 (a) UV-vis absorption spectra of FAP $(20 \mu \mathrm{M})$ in the absence or presence of FA (1 mM) in PBS buffer $(10 \mathrm{mM}$, pH 7.4, containing $1 \%$ MeCN) at $37^{\circ} \mathrm{C}$ for $120 \mathrm{~min}$. (b) Fluorescence spectra of FAP $(20 \mu \mathrm{M})$ in the absence or presence of FA $(1 \mathrm{mM})$ in PBS buffer $(10 \mathrm{mM}, \mathrm{pH} 7.4, \mathrm{containing} 1 \%$ $\mathrm{MeCN}$ ) at $37^{\circ} \mathrm{C}$ for $120 \mathrm{~min}$, with excitation at $405 \mathrm{~nm}$, and excitation and emission slit widths $=5 \mathrm{~nm}$.

To assess the response time of FAP towards FA, a solution of FAP $(20 \mu \mathrm{M})$ was added to FA $(2 \mathrm{mM})$ in PBS buffer $(10 \mathrm{mM}, \mathrm{pH}$ 7.4 , containing $1 \% \mathrm{MeCN}$ ) for different incubation times and then measured by a fluorescence spectrometer (Fig. S4 $\dagger$ ). Importantly, a significant ratiometric change was observed even after $5 \mathrm{~min}$, accompanying a pronounced enhancement of the fluorescence intensity at $570 \mathrm{~nm}$ and a distinct reduction at $495 \mathrm{~nm}$, whereas the emission band at $570 \mathrm{~nm}$ was gradually enhanced with the extension of the incubation time even after $180 \mathrm{~min}$. Specifically, the fluorescence ratio $I_{570} / I_{495}$ increased by 9 -fold upon reaction with FA for $120 \mathrm{~min}$. In order to observe the relatively obvious fluorescence changes at an appropriate incubation time, we chose a reaction time of $120 \mathrm{~min}$ to further investigate the $\mathrm{pH}$ effect on the probe, FAP. We measured the fluorescence signal ratios of $I_{570} / I_{495}$ towards FA $(2 \mathrm{mM})$ in PBS buffer with various pHs ranging from 3.0 to 11.0 and, surprisingly, found that FAP shows a relatively wide range of $\mathrm{pH}$ application (Fig. S5 $\dagger$ ). Importantly, the remarkable ratiometric fluorescence response and the wide range of $\mathrm{pH}$ application of FAP are advantageous to minimizing the interference of the fluorescence background and increasing the fidelity of the detection signal.

Encouraged by these results, we sought to investigate the sensitivity of FAP for FA, and we assessed the fluorescence response of FAP $(20 \mu \mathrm{M})$ with different concentrations of FA ( 0 $3 \mathrm{mM}$ ) in PBS buffer at $37{ }^{\circ} \mathrm{C}$. The incubation of FAP with increasing concentrations of FA resulted in a gradual increase of the emission intensity at $570 \mathrm{~nm}$ and a corresponding decrease at $495 \mathrm{~nm}$ (Fig. 2 and S6†). Strikingly, an excellent linear dependence $\left(R^{2}=0.98827\right)$ between the fluorescence signal ratios of $I_{570} / I_{495}$ and the concentrations of FA $(0-200 \mu \mathrm{M})$ was observed. Moreover, to further evaluate the validity and stability of the slope, different concentrations of FA $(1.5,4.5$ and 9.5 equivalent) were spiked into the PBS buffer and incubated with FAP $(20 \mu \mathrm{M})$ for $120 \mathrm{~min}$ (Fig. S7 $\dagger)$. After a calculation using a calibration curve, seen in Fig. 2b, the concentration of formaldehyde was determined, and the resultant FA recoveries ranged from $98 \%$ to $106 \%$, revealing that FAP has potential capability for quantitatively monitoring FA. The limit of detection (LOD) of FAP for the detection of FA was $0.5 \mu \mathrm{M}$ in aqueous solution based on the signal-to-noise ratio $(\mathrm{S} / \mathrm{N}=3)$.

The selectivity of the fluorescent probe is an important parameter for assessing if it is suitable for biological application. To determine whether FAP could detect FA specifically, we then examined the fluorescence signal and compared the ratio values of the fluorescence intensity at $570 \mathrm{~nm}$ to $495 \mathrm{~nm}\left(I_{570}\right)$ $I_{495}$ ) after incubation with a series of comparative analytes, including benzaldehyde, methylglyoxal, oxalaldehyde, GSH, $\mathrm{H}_{2} \mathrm{O}_{2}$, amino acids, etc. As seen in Fig. $2 \mathrm{c}$ and $\mathrm{S} 8, \uparrow$ the largest change of the fluorescence signal ratio was observed upon the addition of FA, while the addition of 100 equiv. of the other analytes displayed a negligible response. FAP displayed a 9-13fold greater response toward FA than the other analytes (Fig. 2c). Additionally, exposing FAP to a mixture of FA and other species still yielded a significantly increased fluorescence ratio $\left(I_{570} / I_{495}\right)$ (Fig. S9 $\dagger$ ). These results clearly indicate that FAP exhibits excellent selectivity for FA over other analytes. Collectively, these selective and sensitive assays unambiguously suggest that FAP has potential capability for tracking FA in environmental and biological samples.

Having established FAP determination, we next moved on to evaluate the practicability of FAP. We initially carried out experiments using real-water samples from the Changjiang River, as well as soft drink and rice water samples. FA (2 mM) was previously spiked into these real-water samples and incubated with FAP $(20 \mu \mathrm{M})$ for 2 hours (Fig. 2 d). Obvious changes of the fluorescence signal ratio were observed after incubation of FA in these buffer systems, while the soft drink and rice water samples only showed relatively moderate changes that might be because FA was partially consumed via the interaction with biomolecules. But fortunately, these changes of the fluorescence signal ratio were enough to help us achieve the determination of FA in the real-water samples. Moreover, we prepared dried FA test papers by dipping paper into a FAP solution and further evaporating to remove the solvent (Fig. 2d inset). Interestingly, using this simple FA test paper to detect FA 


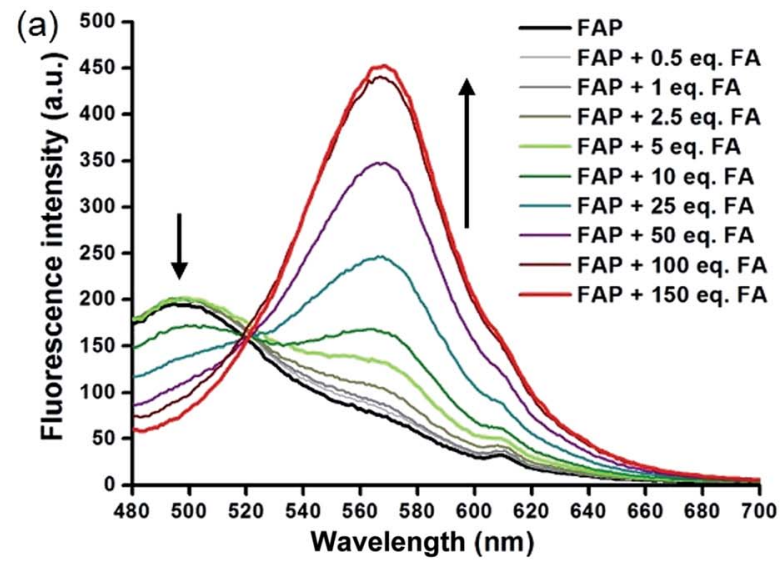

(c)

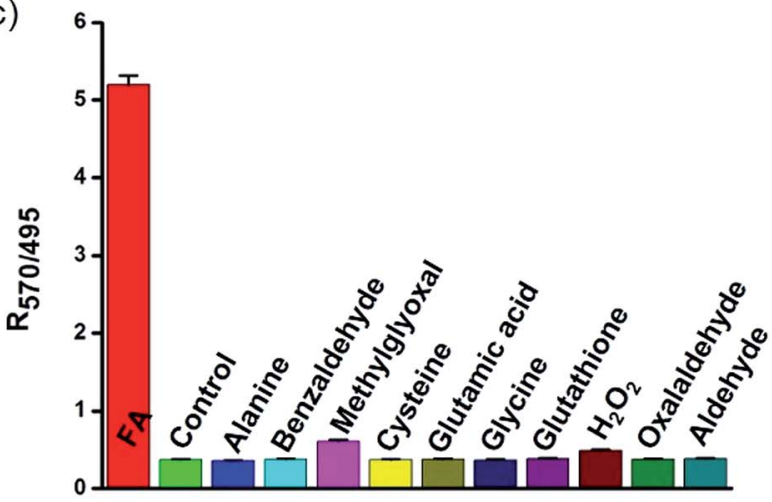

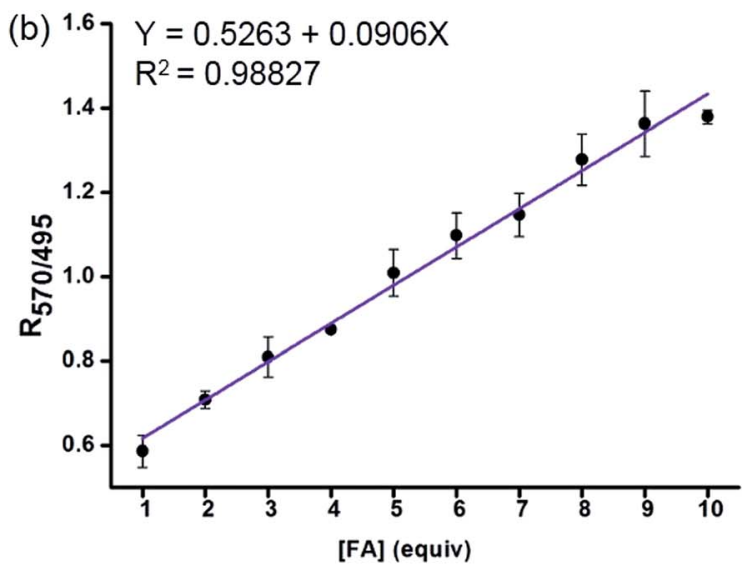

(d)

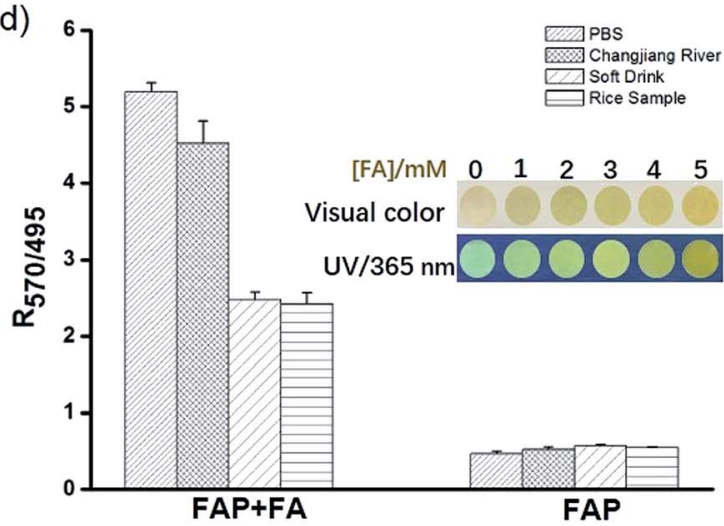

Fig. 2 (a) Fluorescence spectra of FAP $(20 \mu \mathrm{M})$ in PBS buffer (10 mM, pH 7.4, containing 1\% MeCN) after treatment with FA (0-3 mM) for 120 min. (b) The linear relationship between the fluorescence intensity ratio $\left(/_{570} / /_{495}\right)$ and the concentration of FA $(0-10$ equiv. $)$ in PBS buffer $(10 \mathrm{mM}$, pH 7.4 , containing $1 \% \mathrm{MeCN})$ for $120 \mathrm{~min}$. (c) Fluorescence intensity ratios $\left(/_{570} / /_{495}\right)$ of the probe, $\operatorname{FAP}(20 \mu \mathrm{M})$, in the presence of various relevant analytes (100 equiv.) in PBS buffer $(10 \mathrm{mM}, \mathrm{pH} 7.4$, containing $1 \% \mathrm{MeCN})$ at $37^{\circ} \mathrm{C}$. All the fluorescence data were observed after $120 \mathrm{~min}$. $\lambda_{\text {ex }}=$ $405 \mathrm{~nm}$, slit: $5 \mathrm{~nm} / 5 \mathrm{~nm}$. (d) Fluorescence intensity ratio $\left(/ 570 / /_{495}\right)$ changes of FAP $(20 \mu M)$ upon the addition of FA (2 mM) in different buffer systems, including PBS, Changjiang River, soft drink, and rice water samples. Inset: the visual colors or visual fluorescence colors of the filter paper strips loaded with FAP in the presence of different concentrations of FA (0-5 mM). All fluorescent images were captured under irradiation by a $365 \mathrm{~nm}$ UV lamp.

solutions with various concentrations exhibited quick color changes from green to yellow within several seconds, indicating the potential utility of FAP as a simple sensor for tracking FA in environmental samples.

To address the capability of FAP for mapping FA in live tissue, we employed FAP to image FA in the live root tip tissue of Arabidopsis thaliana using a confocal fluorescence microscope. As shown in Fig. 3, the root tip tissues of Arabidopsis thaliana were pretreated with FAP $(50 \mu \mathrm{M})$ for $45 \mathrm{~min}$, and subsequently different concentrations of exogenous FA (0 or $3 \mathrm{mM}$ ) were added and incubated for another $120 \mathrm{~min}$. Gratifyingly, FAP can effectively penetrate the cell wall and cell membrane, and can display a bright fluorescence in the green channel and relatively weak fluorescence in the red channel, suggesting that live Arabidopsis thaliana tissues have a low level of intracellular FA activity. ${ }^{12,13}$ Importantly, upon incubation with a typical stress concentration of exogenous FA ( $3 \mathrm{mM})$, a significant change of the fluorescence signal ratio is observed in the ratiometric fluorescence image $\left(F_{\text {red }} / F_{\text {green }}\right)$ (Fig. 3). This indicates that the alteration of the intracellular FA levels in live plant tissues can be reflected by the fluorescence change of FAP. Notably, these FA concentrations fall well within the scope of physiological concentration, treating the condition of formaldehyde stress in plants, and are estimated to be $0.1-10 \mu \mathrm{mol} \mathrm{g}^{-1}$ in the fresh weight of a plant, ${ }^{13}$ and up to $2-8 \mathrm{mM}$ in the external treatment of HCHO stress. ${ }^{14,15,37}$

To further investigate whether FAP can be applied to detect the change of endogenous FA levels in live plant tissues, we pretreated live root tip tissues of Arabidopsis thaliana with $10 \%$ $\mathrm{MeOH}$ stress for $1 \mathrm{~h}$ to induce the production of endogenous $\mathrm{FA}^{38}{ }^{38}$ then incubated with FAP $(50 \mu \mathrm{M})$ for another 3 hours and imaged after washing with PBS (Fig. 4). The endogenous FA freshly formed via $\mathrm{MeOH}$ pretreatment results in an obvious change of the fluorescence signal ratio in the ratiometric fluorescence image compared with the negative control. Furthermore, the stimulation of endogenous FA uptake with $1 \mathrm{mM} o$ phenanthroline, a known inhibitor of formaldehyde dehydrogenase ${ }^{39}$ also yielded a statistically significant increase in the fluorescence ratio (Fig. 4). All of these data suggest that FAP is capable of ratiometric monitoring of endogenous FA activity in 

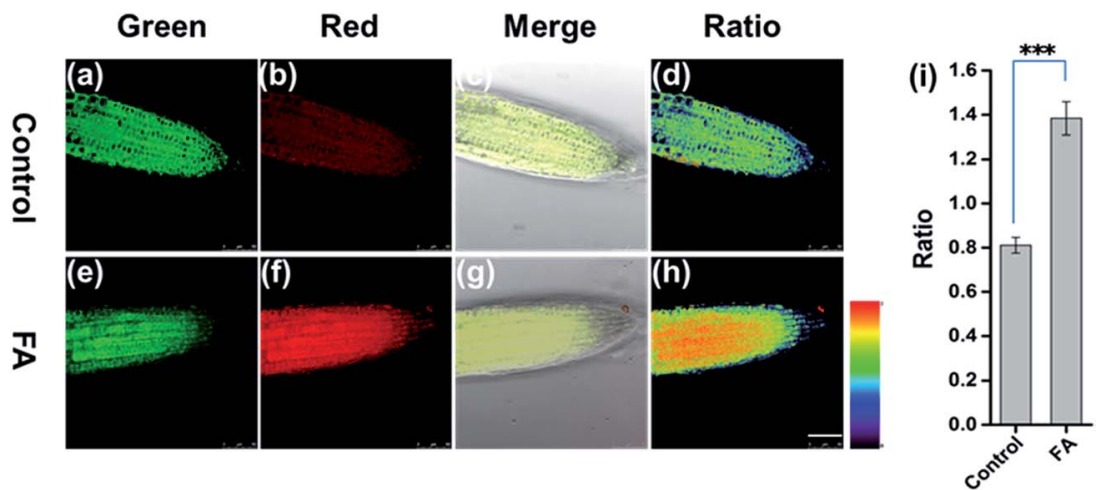

Fig. 3 Confocal microscopy images of Arabidopsis thaliana incubated with FAP (50 $\mu$ M) for 45 min at $37^{\circ} \mathrm{C}$ (a-d), followed by the addition of $3 \mathrm{mM} \mathrm{HCHO}(\mathrm{e}-\mathrm{h})$ and incubation for another 2 hours. (a and e) The green channel $(475-520 \mathrm{~nm})$, (b and f) the red channel (545-595 $\mathrm{nm}$ ), (c and $\mathrm{g}$ ) the merge images of the bright-light field, green channel and red channel, and $\left(\mathrm{d}\right.$ and $\mathrm{h}$ ) the ratio images of the red to green channel. $\lambda_{\text {ex }}=$ $405 \mathrm{~nm}$, scale bar $=50 \mu \mathrm{m}$. (i) Quantification of the imaging data ( $\mathrm{d}$ and h). $n=3$, error bars were \pm SD. Statistical analyses performed with a twotailed Student's $t$-test with unequal variance, $* * * p$ value $<0.0001$

Arabidopsis thaliana in vivo. Finally, we tried to apply FAP to measure the endogenous formaldehyde concentration in the fresh root and leaf tissues of Arabidopsis thaliana. Spiked HCHO was used as the internal standard in the prepared tissue homogenate. The spiked samples were subsequently incubated with the $20 \mu \mathrm{M}$ probe for 2 hours and then monitored (Fig. S10 ${ }^{\dagger}$ ). We found that the concentrations of endogenous formaldehyde in the fresh homogenates of the Arabidopsis thaliana root and leaf tissues are $1.8 \mu \mathrm{mol} \mathrm{g}^{-1}$ and $4.2 \mu \mathrm{mol} \mathrm{g}^{-1}$, respectively. This result is consistent with the previous report that the average endogenous FA concentration was estimated to be $0.1-10 \mu \mathrm{mol} \mathrm{g}{ }^{-1}$ of the fresh weight of the plant. ${ }^{13}$ Taken together, these findings demonstrate that FAP displays the ability of detection of the biologically relevant endogenous formaldehyde in live plant tissues.

\section{Conclusions}

In summary, we have successfully developed a ratiometric fluorescent probe, FAP, for monitoring formaldehyde based on the $N, N$-dimethylquinolin-6-amine fluorophore for the first time. This probe displayed a significant red shift $(75 \mathrm{~nm})$ in the emission profiles and an obvious change of the fluorescence signal ratio for the response of FA. We have demonstrated that this highly selective and effective probe has potential capability for monitoring FA in real-water samples and live plant tissues. In vivo imaging results show that FAP can not only effectively map exogenous FA, but also can sensitively track endogenous FA. To the best of our knowledge, this is the first example of a fluorescent probe-based methodology for the ratiometric imaging of endogenous FA in plant tissues. With these insights,

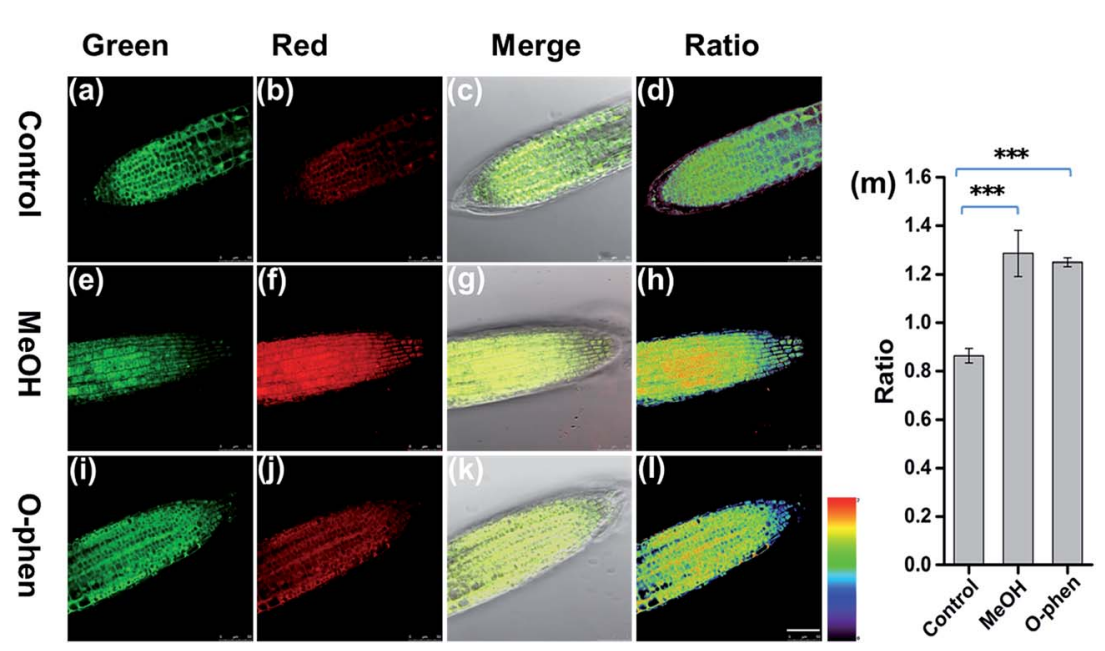

Fig. 4 Confocal microscopy images of endogenous FA in Arabidopsis thaliana via pretreatment with or without $10 \% \mathrm{MeOH}$ and $1 \mathrm{mM}$ ophenanthroline in PBS buffer for $1 \mathrm{~h}$, followed by exchange into fresh PBS and further incubation with $\mathrm{FAP}(50 \mu \mathrm{M})$ for $3 \mathrm{~h}$ at $37^{\circ} \mathrm{C}$ before imaging. (a, e, and i) Green channel (475-520 nm), (b, f, and j) red channel (545-595 nm), and (c, g, and k) merge images of the bright-light field, green channel and red channel, and $(d, h, l)$ the ratio images of the red to green channel. $\lambda_{\text {ex }}=405 \mathrm{~nm}$, Scale bar $=50 \mu \mathrm{m}$. $(\mathrm{m})$ Quantification of the imaging data $(d, h, l) . n=3$, the error bars were \pm SD. Statistical analyses performed with a two-tailed Student's $t$-test with unequal variance, $* * * p$ value $<0.001$ 
we anticipate that the further application of FAP to the fluorescent imaging of FA in other plants will enable better understanding of its metabolism mechanism, thus promoting the progress of FA related physiological and pathological studies.

\section{Acknowledgements}

This work is financially supported by grants from the National Natural Science Foundation of China (21302094), the Jiangsu Natural Science Foundation (BK20130552), the Research Fund for the Doctoral Program of Higher Education of China (20130091120036), the Fundamental Research Funds for the Central Universities (20620140214), and the Scientific Research Foundation of Nanjing Normal University (184080H202B95).

\section{Notes and references}

1 M. A. Flyvholm and P. Andersen, Am. J. Ind. Med., 1993, 24, 533-552.

2 E. A. Hoffman, B. L. Frey, L. M. Smith and D. T. Auble, J. Biol. Chem., 2015, 290, 26404-26411.

3 R. Baan, Y. Grosse, K. Straif, B. Secretan, F. El Ghissassi, V. Bouvard, L. Benbrahim-Tallaa, N. Guha, C. Freeman, L. Galichet and V. Cogliano, Lancet Oncol., 2009, 10, 11431144.

4 Z. Tong, C. Han, W. Luo, X. Wang, H. Li, H. Luo, J. Zhou, J. Qi and R. He, Age, 2013, 35, 583-596.

5 M. Hauptmann, P. A. Stewart, J. H. Lubin, L. E. Beane Freeman, R. W. Hornung, R. F. Herrick, R. N. Hoover, J. F. Fraumeni Jr, A. Blair and R. B. Hayes, J. Natl. Cancer Inst., 2009, 101, 1696-1708.

6 P. H. Yu, J. Neural Transm., Suppl., 1998, 52, 201-216.

7 M. Giese, U. Bauer-Doranth, C. Langebartels and H. Sandermann Jr, Plant Physiol., 1994, 104, 1301-1309.

8 H. Achkor, M. Diaz, M. R. Fernandez, J. A. Biosca, X. Pares and M. C. Martinez, Plant Physiol., 2003, 132, 2248-2255.

9 A. D. Hanson and S. Roje, Annu. Rev. Plant Physiol. Plant Mol. Biol., 2001, 52, 119-137.

10 H. Heck and M. Casanova, Regul. Toxicol. Pharmacol., 2004, 40, 92-106.

11 S. Kato, P. J. Burke, T. H. Koch and V. M. Bierbaum, Anal. Chem., 2001, 73, 2992-2997.

12 G. Blunden, B. G. Carpenter, M. Adrian-Romero, M. H. Yang and E. Tyihak, Acta Biol. Hung., 1998, 49, 239-246.

13 L. Trezl, L. Hullan, T. Szarvas, A. Csiba and B. Szende, Acta Biol. Hung., 1998, 49, 253-263.

14 R. Wang, Z. Zeng, T. Liu, A. Liu, Y. Zhao, K. Li and L. Chen, Plant Physiol. Biochem., 2016, 105, 233-241.

15 L. M. Chen, H. Yurimoto, K. Z. Li, I. Orita, M. Akita, N. Kato, Y. Sakai and K. Izui, Biosci., Biotechnol., Biochem., 2010, 74, 627-635.

16 S. Zhou, S. Xiao, X. Xuan, Z. Sun, K. Li and L. Chen, Plant Physiol. Biochem., 2015, 89, 53-63.
17 T. F. Brewer and C. J. Chang, J. Am. Chem. Soc., 2015, 137, 10886-10889.

18 A. Roth, H. Li, C. Anorma and J. Chan, J. Am. Chem. Soc., 2015, 137, 10890-10893.

19 Y. Tang, X. Kong, A. Xu, B. Dong and W. Lin, Angew. Chem., Int. Ed. Engl., 2016, 55, 3356-3359.

20 Y. H. Lee, Y. Tang, P. Verwilst, W. Lin and J. S. Kim, Chem. Commun., 2016, 52, 11247-11250.

21 J. B. Li, Q. Q. Wang, L. Yuan, Y. X. Wu, X. X. Hu, X. B. Zhang and W. Tan, Analyst, 2016, 141, 3395-3402.

22 Y. Qian, M. Schurmann, P. Janning, C. Hedberg and H. Waldmann, Angew. Chem., Int. Ed. Engl., 2016, 55, 77667771.

23 T. Liu, J. Lin, Z. Li, L. Lin, Y. Shen, H. Zhu and Y. Qian, Analyst, 2015, 140, 7165-7169.

24 Y. Qian, J. Karpus, O. Kabil, S. Y. Zhang, H. L. Zhu, R. Banerjee, J. Zhao and C. He, Nat. Commun., 2011, 2, 495.

25 S. Bhuniya, S. Maiti, E. J. Kim, H. Lee, J. L. Sessler, K. S. Hong and J. S. Kim, Angew. Chem., Int. Ed. Engl., 2014, 53, 44694474.

26 J. J. Hu, N. K. Wong, S. Ye, X. Chen, M. Y. Lu, A. Q. Zhao, Y. Guo, A. C. Ma, A. Y. Leung, J. Shen and D. Yang, J. Am. Chem. Soc., 2015, 137, 6837-6843.

27 K. Xu, D. Luan, X. Wang, B. Hu, X. Liu, F. Kong and B. Tang, Angew. Chem., Int. Ed. Engl., 2016, 55, 12751-12754.

28 X. Wu, L. Li, W. Shi, Q. Gong and H. Ma, Angew. Chem., Int. Ed. Engl., 2016, 55, 14728-14732.

29 Y. Tang, X. Kong, Z. R. Liu, A. Xu and W. Lin, Anal. Chem., 2016, 88, 9359-9363.

30 Z. Guo, G. H. Kim, J. Yoon and I. Shin, Nat. Protoc., 2014, 9, 1245-1254.

31 J. Fan, M. Hu, P. Zhan and X. Peng, Chem. Soc. Rev., 2013, 42, 29-43.

32 C. Huang, T. Jia, M. Tang, Q. Yin, W. Zhu, C. Zhang, Y. Yang, N. Jia, Y. Xu and X. Qian, J. Am. Chem. Soc., 2014, 136, 1423714244.

33 L. He, X. Yang, Y. Liu, X. Kong and W. Lin, Chem. Commun., 2016, 52, 4029-4032.

34 C. G. Goodman and J. S. Johnson, J. Am. Chem. Soc., 2015, 137, 14574-14577.

35 L. Xue, G. Li, D. Zhu, Q. Liu and H. Jiang, Inorg. Chem., 2012, 51, 10842-10849.

36 Q. Q. Wu, Z. F. Xiao, X. J. Du and Q. H. Song, Chem.-Asian J., 2013, 8, 2564-2568.

37 Z. B. Song, S. Q. Xiao, L. You, S. S. Wang, H. Tan, K. Z. Li and L. M. Chen, Plant, Cell Environ., 2013, 36, 1490-1506.

38 A. Downie, S. Miyazaki, H. Bohnert, P. John, J. Coleman, M. Parry and R. Haslam, Phytochemistry, 2004, 65, 23052316.

39 S. Ogushi, M. Ando and D. Tsuru, J. Biochem., 1984, 96, 15871591. 